\title{
Afección coronaria en niños con enfermedad de Kawasaki
}

\author{
Cristina G Battagliotti ${ }^{1}$, Mario Karakachoff ${ }^{1}$, Francisco Gonzalez ${ }^{1}$, Inés Martinez ${ }^{1}$ \\ ${ }^{1}$ Servicio de Reumatología y Cardiología Hospital de Niños Dr. Orlando Alassia, Mendoza 4151, Santa Fe.
}

Todos los autores mencionados han participado en la contribución del diseño, en la recolección de los datos, en el análisis e interpretación de los mismos.

El trabajo ha sido revisado por el Comité de Docencia del Hospital de Niños Dr. Orlando Alassia.

Han participado en la revisión crítica de su contenido intelectual y han aprobado la versión final del manuscrito. La redacción del mismo a cargo del primer autor.

\section{Resumen}

Objetivo: La enfermedad de Kawasaki (EK) es una vasculitis frecuente en la infancia que puede producir aneurismas coronarios. Con el objetivo de describir las lesiones coronarias tanto en fase aguda como al año del diagnóstico, realizamos este estudio retrospectivo de 30 niños de 0 a 7 años con EK atendidos en nuestro centro, desde enero de 1990 a febrero de 2010.

Resultados: Presentaron afección coronaria 7 pacientes. Cuatro de ellos varones. El 71,4\% (n: 5) tenían EK completo. La edad media de inicio de los síntomas fue de 1,55 años (rango: 0,083-3). La mayoría de las lesiones fueron menores tales como la ectasia difusa o el aneurisma pequeño de ambas coronarias (n: 5). Estables al año de seguimiento. Dos varones de 19 días y 7 meses de edad presentaron lesiones mayores que correspondieron a los aneurismas múltiples y un aneurisma gigante persistente. La duración de la fiebre $(p=0,04)$ y el retraso de aplicación de la gammaglobulina $(p=0,025)$ pudo asociarse con afección coronaria.

Conclusión: Alto índice de coronariopatías. La mayoría de las lesiones fueron menores tales como las ectasias difusas o los aneurismas pequeños estables al año de evolución. Las lesiones mayores correspondieron a los aneurismas múltiples y un aneurisma gigante persistente. La duración de la fiebre y el retraso en el tratamiento se asoció con mayor compromiso coronario. Recomendamos al médico mantener un alto índice de sospecha de esta enfermedad particularmente en los niños menores de un año de vida.

Palabras clave: enfermedad de Kawasaki, aneurisma coronario.

\section{Summary}

Objective: Kawasaki's disease (KD) is a systemic vasculitis of childhood with a predilection for the coronary arteries. The aim of this study was to describe the coronary lesions of patients with KD both in the acute phase and after one year. A retrospective study was performed on 30 children with $\mathrm{KD}$ from 0 to 7 years of age treated in our hospital between January 1990 and February 2010.

Results: Coronary artery disease was recorded in 7 patients. Median age at diagnosis was 1.55 years (range: $0.083-3$ ). There were four boys, $71.4 \%$ (n: 5), with typical disease. Most injuries were minor, diffuse ectasia or small aneurysm of both coronary arteries persisted during follow-up at one year. Two males of 19 days and 7 months of age developed multiple aneurysms and giant aneurysm respectively. Duration of fever $(p=0.04)$ and delay of diagnosis and treatment with intravenous gamma globulin $(p=0.025)$ were associated with coronary disease.

Conclusion: We found a high percentage of coronary artery abnormalities. Mainly ectasia and small aneurysms persistent at one Year of follow. Major lesions were multiple aneurysms and giant aneurysm that were associated with delayed diagnosis and treatment. We recommend clinicians to mantain high levels of suspicion, particularly in infants under one year of age.

Key words: Kawasaki disease, coronary artery disease.

\section{Correspondencia}

Autor para envío de notificaciones: Battagliotti, Cristina

E-mail: cristinabattagliotti@yahoo.com.ar 


\section{Introducción}

La enfermedad de Kawasaki (EK) es una vasculitis frecuente en la infancia que puede lesionar las arterias coronarias originando aneurismas que, en ocasiones, se complican con trombosis u obstrucción coronaria provocando isquemia miocárdica ${ }^{1,2}$.

Constituye la primera causa de cardiopatía adquirida en la infancia del adulto joven, desplazando a la Fiebre Reumática en los países desarrollados ${ }^{2,3}$.

Un diagnóstico oportuno y un tratamiento adecuado durante la fase aguda de la enfermedad disminuyen el riesgo de coronariopatía. El uso de gammaglobulina parenteral en los primeros 10 días de la enfermedad disminuye este riesgo de un $20 \%$ a un $5-2 \%$.

El compromiso coronario se presenta con mayor frecuencia en varones, menores de un año, con período de inflamación prolongada o recurrencia de la fiebre luego de un período afebril. También suelen hallarse si el paciente presenta signos de isquemia, afección peri-endo o miocárdica o descenso del nivel plaquetario, albúmina y hematocrito respecto al nivel inicial ${ }^{6,7,8,9,10}$.

Es por esto que nos interesa conocer el daño coronario provocado por la enfermedad en nuestro centro.

\section{Objetivo}

Describir las lesiones coronarias en los pacientes con diagnóstico de EK.

Evaluar la evolución de las mismas al año de enfermedad. Buscar asociaciones para coronariopatía.

\section{Material y métodos}

Se realizó un estudio descriptivo, retrospectivo de los pacientes atendidos en nuestro centro con EK desde enero de 1990 a febrero de 2010.

Se evaluaron 30 pacientes de 0 a 7 años.

La EK fue definida por los criterios de la Asociación Americana de Cardiología (AHA) ${ }^{15}$.

Se considera con EK completa a aquellos pacientes con fiebre mayor o igual a 5 días no explicable por otras causas, y por lo menos 4 de los 5 criterios:

1) Conjuntivitis bilateral no secretora.

2) Cambios en la cavidad oral. Como labios intensamente rojos, secos y resquebrajados (queilitis), o la lengua roja en frutilla, aframbuesada por hipertrofia de las papilas (glositis), y o la faringe congestiva.

3) Exantema polimorfo que involucra el tronco.

4) Cambios en las extremidades como el eritema de palmas y plantas, edema indurado de manos y pies, o descamación de la piel de las manos, pies y o perineal.

5) Linfadenopatía cervical mayor a $1,5 \mathrm{~cm}$, aguda no supurativa.
Se considera con EK incompleta a aquellos con fiebre de más de 5 días con menos de 4 criterios y los atípicos con manifestación inusual pero con compromiso coronario ${ }^{15}$.

Se consideró dilatación coronaria al diámetro mayor de $3 \mathrm{~mm}$ medido por ECO Doppler color, por el mismo equipo y operador para cada paciente en fase aguda y para los afectados al año del diagnóstico. Se clasificaron como ectasia difusa, aneurismas únicos o múltiples, pequeños o medianos hasta $8 \mathrm{~mm}$; aneurismas gigantes mayor de 8 $\mathrm{mm}$ y las lesiones de estenosis coronaria ${ }^{15}$.

Las variables analizadas fueron edad, sexo, duración de la fiebre, número de criterios de la enfermedad, piuria abacteriana, diarrea, irritabilidad, artritis o artralgia, aumento de enzimas hepáticas, nivel inicial de hemoglobina, glóbulos blancos, plaquetas, velocidad de eritrosedimentación globular y tratamiento adecuado en tiempo y forma (gammaglobulina endovenosa $400 \mathrm{mg} / \mathrm{kg} /$ dosis por 4 días o $2 \mathrm{~g} / \mathrm{kg} /$ única dosis más aspirinas $80-100 \mathrm{mg} / \mathrm{kg} / \mathrm{d}$ antes de los 10 días de evolución de la enfermedad).

En el análisis estadístico se usó la prueba de MannWhitney o la prueba exacta de Fisher según corresponda con el programa SPSS versión 16, considerando el valor $\mathrm{p}$ $<0,05$ como significativo.

\section{Resultados}

De un total de 30 pacientes con EK, 7 de ellos presentaron lesión coronaria. Con una edad media de inicio de los síntomas de 1,55 años (rango: 0,083-3), 4 de ellos fueron varones, el 71,4\% (n: 5) tuvieron EK completo.

La Tabla 1 resume las diferencias demográficas y clínicas entre los pacientes con y sin afección coronaria. La Tabla 2 muestra las características de quienes tuvieron afección coronaria (ver Tablas).

Los hallazgos ecocardiográficos más frecuentes fueron dilataciones o ectasia difusas mayor o igual a $3 \mathrm{~mm}$, vistas en 5 pacientes tanto en coronaria derecha como en izquierda, con rangos que oscilaron entre $3,3 \mathrm{~mm}$ a 5,5 $\mathrm{mm}$. Estables al año de seguimiento.

Las lesiones mayores se encontraron en dos varones de 19 días y 7 meses de edad. Ambos niños presentaron período de fiebre prolongada y retardo en la aplicación de la terapéutica con gammaglobulina parenteral, la cual se realizó a los 17 días de iniciada la fiebre.

El primer caso presentó múltiples aneurismas en coronaria derecha, entre $5,9 \mathrm{~mm}$ y $6,7 \mathrm{~mm}$, y dilatación uniforme en coronaria izquierda con dos aneurismas: uno a nivel del ostium de $6 \mathrm{~mm}$ y otro en la descendente anterior de $7 \mathrm{~mm}$ (Figura 1). En el control al año desaparecieron los aneurismas de la coronaria derecha.

El segundo caso se observó con aneurisma gigante mayor a $9 \mathrm{~mm}$ en coronaria derecha y aneurismas múltiples en coronaria izquierda (Figura 2). Al año persistió el aneurisma gigante. 


\begin{tabular}{|c|c|c|c|}
\hline \multirow[b]{2}{*}{ HALLAZGOS CLÍNICOS } & \multicolumn{2}{|c|}{ Grupo } & \\
\hline & $\begin{array}{c}\text { Con lesión } \\
\text { coronaria }(A) n=7\end{array}$ & $\begin{array}{c}\text { Sin coronariopatía } \\
\text { (B) } n=23\end{array}$ & \\
\hline Sexo (\% de varones) & $4 ;(57,1 \%)$ & $10 ;(43,5 \%)$ & 0,53 \\
\hline Edad (años) & $\begin{array}{c}\text { Promedio: } 1,55 \\
\text { Mediana: } 1,58 \\
\text { S: } 1,04 \\
\text { R:(0,083-3) }\end{array}$ & $\begin{array}{c}\text { Promedio: } 2,65 \\
\text { Mediana: } 2,08 \\
\text { S: } 1,99 \\
\text { R:(0,063-7,83) }\end{array}$ & $0,21^{*}$ \\
\hline Época del año & $3 ; 42,9 \%$ verano & $10 ; 43,5 \%$ primavera & -- \\
\hline Días de fiebre & Promedio: 10,71 & Promedio: 6,74 & $0,04^{\star}$ \\
\hline Criterios clínicos ( $\geq 4)$ & $5 ;(71,4 \%)$ & $16 ;(69,4 \%)$ & 0,66 \\
\hline Exantema & $7 ;(100 \%)$ & $21 ;(91,3 \%)$ & 0,58 \\
\hline Cambio en extremidades & $7 ;(100 \%)$ & $23 ;(100 \%)$ & -- \\
\hline Compromiso bucal & $7 ;(100 \%)$ & $23 ;(100 \%)$ & -- \\
\hline Inyección conjuntival & $5 ;(71,4 \%)$ & $14 ;(60,9 \%)$ & 0,49 \\
\hline Adenomegalia cervical & $1 ;(14,2 \%)$ & $5 ;(21,7 \%)$ & 0,57 \\
\hline \multicolumn{4}{|l|}{ Otras manifestaciones } \\
\hline Piuria & 3 & 8 & 0,51 \\
\hline Atralgia / Artritis & 4 & 3 & 0,03 \\
\hline Aumento de TGP y TGO & 5 & 7 & 0,07 \\
\hline Irritabilidad & 2 & 1 & 0,13 \\
\hline Diarrea & 3 & 3 & 0,12 \\
\hline VSG $>=20$ & 6 & 19 & 0,67 \\
\hline Leucocitos $>=10.000$ & 5 & 17 & 0,62 \\
\hline Anemia $\mathrm{Hb}<=10$ & 4 & 9 & 0,34 \\
\hline Plaquetas $>=200.000$ & 6 & 21 & 0,56 \\
\hline
\end{tabular}

Tabla 1. Hallazgos clínicos y de laboratorio comparativo entre pacientes con lesión coronaria y sin coronariopatía.

Dentro del grupo no afectado hubo 3 casos con hiperrefringencia coronaria transitoria.

En nuestra población, la duración de la fiebre $(\mathrm{p}=0,04)$, la presencia de artritis $(\mathrm{p}=0,03)$ y el retardo en la aplicación de la gammaglobulina $(\mathrm{p}=0,025)$ se asoció con mayor riesgo de lesión.

\section{Discusión}

Las vasculitis son un conjunto de enfermedades multisistémicas que comparten la lesión histopatológica de la inflamación de la pared vascular. La púrpura de Shonlein Henoch junto con la EK constituye una de las vasculitis más frecuente en pediatría ${ }^{11}$.

La EK es una vasculitis sistémica aguda que compromete vasos de mediano calibre, autolimitada, de origen desconocido, que afecta sobre todo a niños pequeños, siendo particularmente grave en los lactantes menores de un año ${ }^{12}$.

Es frecuente en menores de 5 años. Raramente se presenta en neonatos, adolescentes y adultos ${ }^{6}$.

El $86 \%$ de nuestra población fueron menores de 5 años, lo llamativo fue el debut de la enfermedad en un varón de 19 días de vida.

Moruno Tirado describe también un caso en un neonato de 19 días con afección cardíaca muy severa. Hasta ese momento solo se habían publicado 10 casos en menores de 30 días, en su mayoría con presentación atípica de difícil diagnóstico y cardiopatías severas ${ }^{13,14}$.

No encontramos un predominio por sexo ni estacional. La mayoría de los casos correspondieron a formas completas de la enfermedad a diferencia de lo que señalan otros autores ${ }^{2,7,8}$.

Un alto porcentaje de pacientes, $23,3 \%$, tuvieron afección coronaria.

Observamos que los niños fueron derivados en forma tardía por cuadros febriles no resueltos. No recibieron gammaglobulina o lo hicieron después del décimo día de evolución. Esta tendencia se revierte en los últimos años.

Royle y otros ${ }^{8}$ también reportan un alto compro-

\begin{tabular}{|c|c|c|c|c|c|c|}
\hline Sexo & Edad & (IVIG < 10 dias) & Días de fiebre & Criterios clínicos & Afección coronaria & Evolución al año \\
\hline $\mathrm{F}$ & 3a & No & 10 & 5 & $\begin{array}{l}\text { CD: } 3 \mathrm{~mm} \\
\text { Cl: } 4 \mathrm{~mm} \\
\text { CD: } 3,9 \mathrm{~mm}\end{array}$ & $\begin{array}{l}\text { CD: } 3 \mathrm{~mm} \\
\text { Cl: } 3 \mathrm{~mm} \\
\text { CD: } 3 \mathrm{~mm}\end{array}$ \\
\hline M & $19 m$ & No & 6 & 4 & $\begin{array}{c}\text { Cl: } 4 \text { mm } \\
\text { CD: am 5,9 mm - } 7 \text { mm }\end{array}$ & $\begin{array}{l}\text { Cl: } 4 \mathrm{~mm} \\
\text { CD: } 3 \mathrm{~mm}\end{array}$ \\
\hline M & $19 d$ & No & 17 & 4 & $\begin{array}{l}\text { Cl: } 6 \mathrm{~mm} \text { y } 7 \mathrm{~mm} \\
\text { CD: } 3,9 \mathrm{~mm}\end{array}$ & $\begin{array}{l}\text { Cl: } 5 \mathrm{~mm} \\
\text { CD: } 4,7 \mathrm{~mm}\end{array}$ \\
\hline $\mathrm{F}$ & $14 \mathrm{~m}$ & No & 10 & 4 & $\begin{array}{l}\mathrm{Cl}: 3,8 \mathrm{~mm} \\
\text { CD: } 3 \mathrm{~mm}\end{array}$ & $\begin{array}{l}\text { Cl: } 4,2 \mathrm{~mm} \\
\text { CD: } 2 \mathrm{~mm}\end{array}$ \\
\hline $\mathrm{F}$ & $2 \mathrm{a} 7 \mathrm{~m}$ & No & 12 & 3 & $\begin{array}{c}\text { Cl: } 4,7 \mathrm{~mm} \\
\text { CD: a. gigante } 9 \mathrm{~mm}\end{array}$ & $\begin{array}{l}\text { Cl: } 3 \mathrm{~mm} \\
\text { CD: a. } 7 \mathrm{~mm}\end{array}$ \\
\hline M & $7 \mathrm{~m}$ & No & 17 & 4 & $\begin{array}{l}\text { Cl: aneurismas múltiples } \\
\text { CD: } 4,8 \mathrm{~mm}\end{array}$ & $\begin{array}{l}\text { Cl: } 3 \mathrm{~mm} \\
\text { CD: } 4,8 \mathrm{~mm}\end{array}$ \\
\hline $\mathrm{F}$ & $1 \mathrm{a} 10 \mathrm{~m}$ & Sí & 6 & 4 & Cl: $4,3 \mathrm{~mm}$ & Cl: $4,3 \mathrm{~mm}$ \\
\hline
\end{tabular}

Tabla 2. Hallazgos en pacientes con afección coronaria. 


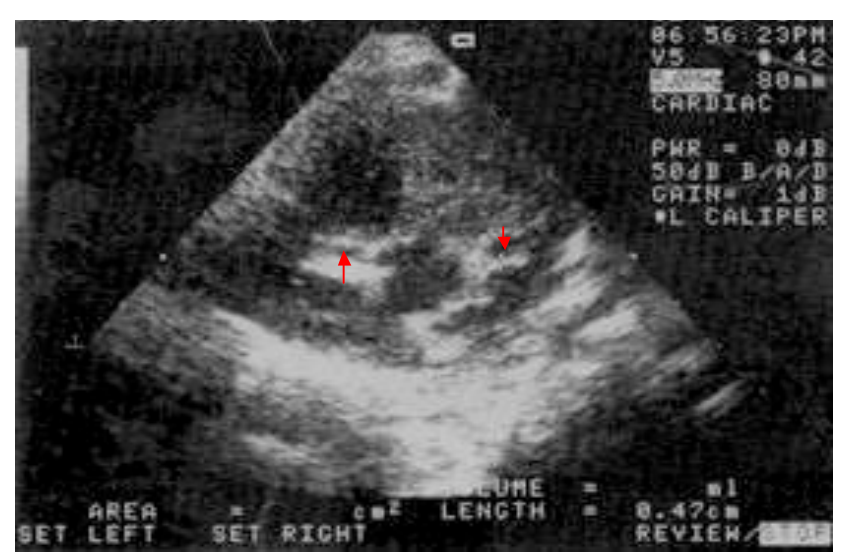

Figura 1. Ecocardiograma de un varón de 19 días que muestra múltiples aneurismas en coronaria derecha tipo arrosariadas y dilatación uniforme de coronaria izquierda.

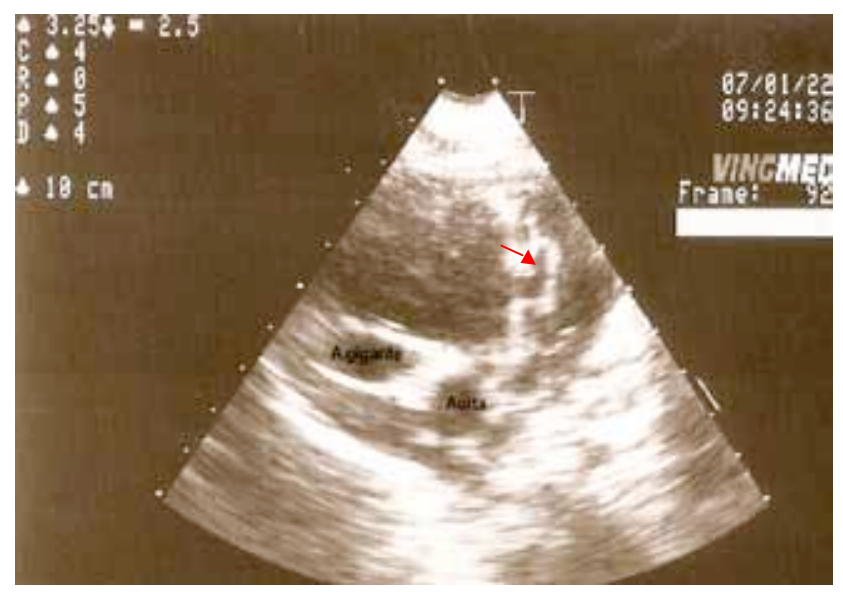

Figura 2. Ecocardiograma de varón de 7 meses. Nótese un aneurisma gigante de más de $9 \mathrm{~mm}$ en coronaria derecha casi del tamaño de la aorta, la flecha señala las lesiones tipo arrosariadas en coronaria izquierda.

miso coronario vinculado al retardo del diagnóstico y tratamiento en la población australiana. Muchos niños presentaban solo algunos criterios diagnósticos en los primeros días de la enfermedad y estos se iban agregando en forma secuencial en el transcurso del tiempo lo que retardaba el diagnóstico.

Es posible que otros factores jueguen un rol importante en el desarrollo del aneurisma. Además de los Criterios de Harada ${ }^{4}$, que sugirió un set de alto riesgo para facilitar la decisión del uso de gammaglobulina parenteral altas dosis, numerosos datos se han relacionado con la aparición de aneurisma coronario, la elevación de la VSG o PCR, leucocitosis $>12.000 \mathrm{ug} / \mathrm{l}$, plaquetas $<350.000 \mathrm{ug} / \mathrm{l}$, niveles bajos de hemoglobina, hematocrito $<35 \%$, albúmina sérica disminuida, elevación de transaminasas, factor de necrosis tumoral alfa o g, interleuquinas 6 y 8 , e hiponatremia $9,10,15,16,17,18$.

La ectasia coronaria es la lesión más frecuente vista en la etapa aguda de la enfermedad que tiende a involucionar en el transcurso de los primeros años ${ }^{17}$.

También en nuestros pacientes la mayoría de las lesiones fueron menores de ectasia difusa o aneurisma pequeño. Dos pacientes tuvieron lesiones mayores como los aneurismas múltiples o aneurisma gigante.

Alrededor del 1\% de los aneurismas pueden llegar a ser gigantes, lo que complica el pronóstico pudiendo generar obstrucción, isquemia miocárdica e incluso rupturas de las coronarias. Koyanagi y otros ${ }^{17}$ establecieron como factores de riesgo para aneurisma gigante la elevación de la PCR, los bajos niveles de potasio y la edad menor de 12 meses.

Mueller y otros ${ }^{18}$ en el seguimiento de las lesiones coronarias encontraron que los pacientes con aneurismas de más de $5 \mathrm{~mm}$ de diámetro detectados en fase aguda desarrollaban una severa estenosis del extremo proximal o distal, requiriendo una intervención quirúrgica con una media de un intervalo de 9,8 años.

Se reporta que un $50 \%$ de las anomalías coronarias regresan a los 3 años de seguimiento mientras que los aneurismas medianos se resuelven dentro de los 2 años $^{17,18}$.

A pesar de la resolución de las lesiones, el riesgo de oclusión trombótica y muerte súbita permanece, ya que algunos pacientes con una involución total de los aneurismas sufrieron alteraciones funcionales y arteriosclerosis prematura de las arterias previamente comprometidas ${ }^{19}$.

Las ventajas de este trabajo fue conocer el daño coronario en nuestra población, el cual se presentó en su mayoría en niños menores vinculados a la duración de la fiebre y al retardo en la aplicación del tratamiento adecuado. Pero tiene sus limitaciones al ser retrospectivo y con un bajo número de casos.

Recomendamos al médico mantener un alto índice de sospecha de esta enfermedad, particularmente en los niños menores de un año de vida.

\section{Conclusión}

Alto índice de lesiones coronarias en pacientes menores con períodos de fiebre prolongado y retraso del tratamiento. La mayoría de ellas ectasias difusas, estables al año. Otras aneurismas múltiples y gigante persistente.

\section{Agradecimientos}

Los autores agradecen a la ingeniera Liliana Contini y a la licenciada Elena Carrera, del Departamento de Matemáticas de la UNL, por su colaboración en el análisis de los datos estadísticos. 


\section{Bibliografía}

1. Kawasaki T, Kosaki F, Okawa S, Shigematsu I, Yanagawa $\mathrm{H}$. A new infantile febrile mucocutaneaus lymph node syndrome prevailing in Japan. Pediatrics 1974; 54:271-6.

2. Anderson MS, Todd JK, Glodé MP. Delayed diagnosis of Kawasaki Syndrome: an analysis of the problem. Pediatrics 2005; 115:428-433.

3. Senzaki H. Long-term outcame of Kawasaki Disease. Circulation 2008; 118:2763-72.

4. Harada K. Intravenous gamma-globulin treatment in Kawasaki disease. Acta Pediatr. Jpn 1991; 33:80510.

5. Moreno N, Mendez-Echeverría A, Inocencio J, Del Castillo F, Baquero-Artigao F, García Miguel M, De José M, Aracil J. Coronary Involvement in Infants with Kawasaki Disease Treated with Intravenous y-Globulin. Pediatr Cardiol. 2008; 29:31-35.

6. Rosenfeld EA, Corydon KE, Shulman ST. Kawasaki disease in infants less than one year of age. J Pediatr.1995; 126:524-9.

7. Schroh A, Dominguez P, Laghezza L, Melonari P, Olguín M, Miatello R. Enfermedad de Kawasaki: afección cardíaca durante la infancia. Rev Esp Cardiol. 2006; 59:387-390.

8. Royle J, Burgner D, Curtis N. The diagnosis and management of Kawasaki disease. J Pediatr Child Health 2005; 41:87-93.

9. Koren G, Lavi S, Rose V, Rowe R. Kawasaki disease: Review of risk factors for coronary aneurysms. J Pediatr 1986; 108:388-392.

10. Fukunihi M, Kikkawa M, Humanana K, Onodera T, Matsuzaki K, Matsumoto Y, Hara J. Prediction of non-responsiveness to intravenous high-dose yglobulin therapy in patients with Kawasaki disease at onset J Pediatr. 2000; 137:172.

11. Katsicas M, Battagliotti C, Russo R. Vasculitis en el niño. En: Battagliotti CA, Berbotto G, Kilstein J, Pons-Estel B. Vasculitis Sistémicas. Toma de decisiones. Buenos Aires Corpus Editora 2013; 311-329.

12. Cuttica R. Enfermedad de Kawasaki. En: Battagliotti CA, Berbotto G, Kilstein J, Pons-Estel B. Vasculitis Sistémicas. Toma de decisiones. Buenos Aires Corpus Editora 2013; 112-119.
13. Moruno Tirado A, Grueso Montero J, Macías Díaz C, Zamarriego Zubizarreta C, Santos de Soto J. Enfermedad de Kawasaki neonatal grave. An Pediatr (Barc). 2007; 67:401-2.

14. Stanley TV, Grimwood K. Classical Kawasaki disease in a neonate. Arch Dis Child Fetal Neonatal ed. 2002; 86:35-36.

15. Newburger JW, Takahashi M, Gerber MA, et al. Diagnosis, treatment, and long-term management of Kawasaki disease: a statement for health professionals from the Comité on Rheumatic Fever, Endocarditis, and Kawasaki Disease, Council on Cardiovascular Disease in the Young American Heart Association. Pediatrics 2004; 114:1708-33.

16. Wilder M, Palinkas L, Kao A, Bastian J, Turner C, Burns J. Delayed diagnosis by physicans contributes to the development of coronary artery aneurysms in children with Kawasaki Syndrome. The Pediatr Infect. Dis J. 2007; 26:256-260.

17. Koyanagi H, Nakamura Y, Yanagawa H. Lower level of serum potasium and higer level of C-reactin protein as an independent risk factor for giant aneurysms in Kawasaki disease. Acta Pediatr.1998; 87:32-6.

18. Mueller F, Knirsch W, Harpes P, Pretre R, Buechel VE, Kretschmar O. Long-term follow-up of acute changes in coronary artery diameter caused by Kawasaki disease : risk factors for development of stenotic lesions. Clin Res Cardiol. 2009; 98:501-507.

19. Noto N, Okada T, Yamasuge M, Taniguchi K, Karasawa K, Ayusawa M, Sumitomo N, Harada K. Nonivasive assessment of the early progression of atherosclerosis in adolescents with Kawasaki disease and coronary artery lesions. Pediatrics 2001; 107:1095-1099. 